\title{
Pair production and gravity as the weakest force
}

\author{
Eduardo Gonzalo and Luis E. Ibáñez \\ Departamento de Física Teórica and Instituto de Física Teórica UAM/CSIC, \\ Universidad Autónoma de Madrid, \\ Cantoblanco, 28049 Madrid, Spain \\ E-mail: eduardo.gonzalo@uam.es, luis.ibannez@uam.es
}

ABSTRACT: The Weak Gravity Conjecture (WGC) is usually formulated in terms of the stability of extremal black-holes or in terms of long distance Coulomb/Newton potentials. However one can think of other physical processes to compare the relative strength of gravity versus other forces. We argue for an alternative formulation in terms of particle pair production at threshold or, equivalently, pair annihilation at rest. Imposing that the production rate by any force mediator (photon or scalar) of pairs of charged particles be larger or equal to graviton production, we recover known conditions for the U(1) WGC and its extensions. Unlike other formulations though, threshold pair production is sensitive to short range couplings present in scalar interactions and gives rise to a Scalar WGC. Application to moduli scalars gives rise to specific conditions on the trilinear and quartic couplings which involve first and second derivatives of the WGC particle mass with respect to the moduli. Some solutions saturating equations correspond to massive states behaving like BPS, KK and winding states which feature duality invariance and are in agreement with the Swampland distance conjecture. Conditions for $N=2$ BPS states saturate our bounds and we discuss specific examples of BPS states which become massless at large Kahler moduli in Type IIA N=2, D=4 CY and orbifold compactifications. We study possible implications for potentials depending on moduli only through WGC massive states. For some simple classes of potentials one recovers constraints somewhat similar but not equivalent to a Swampland dS conjecture.

KEYwords: Superstring Vacua, Superstrings and Heterotic Strings

ArXiv ePrint: 2005.07720 


\section{Contents}

1 Introduction 1

2 The PPWGC for U(1) interactions 5

2.1 A single $\mathrm{U}(1) \quad 5$

2.2 Multiple U(1)'s 8

3 Pair production from scalars and the Scalar Weak Gravity Conjecture 10

$\begin{array}{lll}3.1 & \text { Examples } & 12\end{array}$

$\begin{array}{lll}3.1 .1 & \text { Complex scalar in } N=1 \text { supergravity } & 12\end{array}$

$\begin{array}{lll}3.1 .2 & \text { Examples from BPS states in } N=2 \text { supergravity } & 14\end{array}$

$\begin{array}{lll}3.1 .3 & \text { The case of } \mathrm{n} \text { real scalar fields } & 15\end{array}$

$\begin{array}{ll}\text { 3.1.4 Previous formulations of the SWGC } & 16\end{array}$

4 Constraints on the scalar potential for moduli 18

5 Strong Scalar Weak Gravity Conjecture 20

$6 \quad$ Final comments and conclusions $\quad 22$

\section{Introduction}

The first formulations of the Weak Gravity Conjecture (WGC) rested heavily on blackhole physics. The simplest version of the U(1) Weak Gravity Conjecture [1-3] (see [4] for a recent review and references) may be formulated from the kinematic condition that extremal black-holes can decay, which requires that a particle with charge $e$ and mass $m$ must exist such that $\sqrt{2} e \geq m / M_{p}$. This may also be understood as a condition between the strengths of the gravitational and the gauge interactions. The condition corresponds to imposing that, between two particles with identical masses and charges, the gauge repulsion dominates, and no bound states form. So it is reasonable to name this as the Weak Gravity Conjecture. This has been generalized to the case of multiple U(1) interactions, which requires some refinements [5-7]. Thus e.g. for an extremal black-hole to decay, it is not enough that particles with mass $m_{i}$ and charge $e_{i}$ exist with $\sqrt{2} e_{i} \geq m_{i} / M_{p}$ for each $\mathrm{U}(1)$, but instead that a certain condition involving the convex hull is met [5]. Furthermore, if we insist that the constraints remain valid under dimensional reduction, string theory examples have shown us that there must exist a sublattice (or a tower) of infinite superextremal massive charged particles verifying the appropriate generalized version of the constraints [6-9]. These generalized versions of the WGC for multiple U(1)'s have passed by now a number of tests within the context of string theory. 
The situation becomes more complicated in the presence of scalar couplings. Scalar couplings do not carry in general a conserved charge and the most naive arguments based on extremal black-hole stability do not directly apply. Furthermore, the question arises whether the Swampland conditions have to do only with black-hole physics or rather with a fundamental general principle that gravity is always the weakest force. This would imply the wanted instability of extremal black-holes but it may also lead to further constraints on different systems other than black-holes. As we said, for U(1) interactions and in the absence of scalar fields, imposing that long range Coulomb forces dominate over Newton attraction gives equivalent results than instability of extremal black-holes [2, 10]. However, if gravity is the weakest force, the condition should apply not only to gauge couplings but also to scalar and Yukawa couplings. In particular, $d=4$ quartic scalar interactions are short-range and such kind of arguments based on long range forces would yield no information about them. Moreover, since a higher dimensional graviton gives rise to lower dimensional scalar fields, if the principles behind the WGC are to apply in any dimension, then some form of a scalar WGC (SWGC) is expected to exist.

In order to compare the strength of gravity with other interactions we should evaluate amplitudes or rates for some kinematic configuration and fixed specific momenta. In the case at hand there are essentially two ways to evaluate these rates at tree level 1) Through diagrams involving one propagator of the considered massless mediators (photon, graviton, moduli) and 2) Through diagrams involving the exchange of a charged massive test particle (e.g. a BPS state). The first possibility involves only long range interactions and includes the exchange of gravitons and photons. With the massive particles at rest they give rise to Coulomb and Newton potentials in the non-relativistic limit. As we said, one can obtain the U(1) WGC constraint from imposing that Coulomb repulsion dominates. In the class 2) of diagrams it is the massive particles which are exchanged and hence they instead are sensitive to short range interactions. Keeping in parallel with the first class, we consider the massive particles almost at rest. There are three type of tree level processes in this class, which are related by crossing symmetry: a) Pair production of a pair of massive states (e.g. $\gamma \gamma \rightarrow \psi \bar{\psi}$ ), b) annihilation of a pair massive states (e.g. $\psi \bar{\psi} \rightarrow \gamma \gamma$ ) and 3) Compton scattering.

Both classes of processes give rise to complementary information concerning the strength of gravity versus other interactions. In particular the second class, which involves the propagator of a massive state, is sensitive to short distance interactions. Contact interactions exist in $d=4$ for the coupling between gauge bosons and charged scalars, $e^{2} A^{\mu} A_{\mu}|\phi|^{2}$. However this brings no uncertainty in the strength of the interaction, since gauge invariance relates this coupling to the trilinear gauge coupling $e A_{\mu} \phi^{*} \partial^{\mu} \phi$. However, in the case of quartic scalar couplings like $\lambda|\phi|^{2}|H|^{2}$ with e.g. $\phi$ a modulus and $H$ some massive scalar, no information about its strength is in general provided by one-particle exchange diagrams. In fact such quartic interactions are known to exist in examples of BPS states of $N=2$ supergravity [11-13] and hence one would like to take them into account in our understanding of gravity as the weakest force ideas.

In order to compare the strength of some interaction induced by some massless mediator (gauge boson or scalar) to gravitational interactions we propose to use the second 
class of processes involving a massive propagator. In particular we will consider pair production of massive states at threshold. The inverse process, massive particle annihilation at rest would yield equivalent results. In the rest of the paper we will talk mainly about pair production but we must emphasize that all the discussion goes through replacing pair production at threshold by pair annihilation at rest. In such kinematical regimes both trilinear and local quartic interactions (if present) are tested and may be compared with the analogous production mechanism from graviton production. Strictly speaking cross sections vanish at threshold, what we will be comparing is the differential cross sections or rather the square of the amplitudes near threshold. In the case of pair annihilation we would directly compare the annihilation cross sections at rest.

One of the attractive features of this approach is that one can derive the usual WGC constraints from multiple U(1)'s and a new scalar version of the WGC in a unified manner and starting from a single principle. In fact we believe that our proposal gives the first derivation of a scalar WGC from a general underlying principle. Other previous discussion of a SWGC do not follow in such a direct way since in particular both scalars and gravitons lead to attractive interactions at large distances and hence no-bound-state arguments fail in this case. One has to rely on $N=2$ SUGRA identities so that the evidence outside the $N=2$ case becomes weaker. Another reason to consider the production rate at threshold is its possible connection with extremal-black-hole radiation through charge pair production. Or black-hole pair annihilation into photons/gravitons. We leave the study of this possible connection to future work.

A point to note is that ours is a quantum relativistic condition since it involves particle production and interaction rates. This is unlike the case of one photon/graviton exchange with particles at rest which give rise to the classical non-relativistic Coulomb/Newton potentials.

Specifically, the general idea may be formulated in the following terms. Consider a theory with $\mathrm{U}(1)$ gauge interactions or moduli scalar fields coupled to gravity. Our general proposal may be stated as the

Pair Production Weak Gravity Conjecture (PPWGC). For any rational direction in the charge lattice $\vec{Q}$ and for every point in moduli space, there is a stable or metastable particle $M$ of mass $m$ whose pair production rate by gauge or scalar mediators at threshold is larger than its graviton production rate:

$$
\left|T\left(i j \longrightarrow M M^{*}\right)\right|_{t h}^{2} \geq\left|T\left(g g \longrightarrow M M^{*}\right)\right|_{t h}^{2} .
$$

Here $i, j$ denotes either $\mathrm{U}(1)^{n}$ gauge bosons or scalar moduli fields and the subindex th corresponds to threshold. The criteria we propose could also be easily generalized to theories with non-abelian gauge fields but we will not consider that possibility in the present paper. In sections 2 and 3 in this paper the scalars will be consider massless, having in mind moduli fields. In sections 4 and 5 we will discuss possible extensions to the case in which the scalars are massive.

In order to apply this principle to the case of U(1) couplings we have computed the production rates of charged scalars and fermions starting from photons and gravitons. 
Production from gravitons is a non-trivial calculation. Fortunately it may be obtained by crossing symmetry using results for graviton Compton scattering in the literature [14]. The bounds obtained exactly match the results discussed for the WGC in the literature, imposing the instability of extremal charged Reissner-Nordstrom black-holes. We also extend the analysis to the case of multiple U(1)'s and argue for natural extensions, PPWGC versions of the Tower and Sublattice conjectures.

When the mediators are massless scalars one obtains new interesting constraints. In particular one gets a scalar WGC (SWGC) constraint involving both trilinear and quartic scalar couplings. If the inequalities are saturated, one obtains a differential equation involving scalar masses and their first and second derivatives. This equation is closely related to previous formulas found in [11] and [15]. The precise form of the SWGC conditions depends on the metric of the moduli in the effective field theory, but some general properties of the extremal solutions are as follows.

1. In all of the examples we study there are solutions for the massive scalars saturating the bounds which behave at large moduli like BPS-like, KK or winding states with built-in duality symmetries. This is remarkable since in the effective field theory there was no input related neither to extra dimensions, extended objects nor dualities, just diagrammatics of the particles involved. These solutions are consistent with the Swampland Distance conjecture.

2. The constraint disappears as $M_{p} \rightarrow \infty$, unlike other versions of the WGC involving scalars $[15,37]$.

3. The constraint is consistent with Special Kahler Geometry identities of $N=2$ BPS states. We test it further in a class of Type IIA CY vacua in which towers of BPS particles coming from $D p$-branes wrapping even cycles become massless at large Kahler moduli [18-23]. They saturate our bound and feature the above mentioned duality, which in this case corresponds to T-duality.

The obtained bounds apply to massive states corresponding to BPS-like, KK or winding objects. Those are in general very heavy particles with masses of order the Planck scale unless going to extreme limits in moduli space. On the other hand we would like to see whether we can learn something about constraints on light (but not massless) scalars which may have some relevance in particle physics or cosmology. In this direction we briefly discuss two possibilities.

In section 4 we consider the possibility that the potential of scalar fields (like moduli themselves) is a function of the mass of the WGC fields, with the latter subject to the derived bounds. In a simplified case of a single massive object one obtains interesting constraints having some resemblance with the refined dS conjecture of refs. [25-27]. Extrema have constraints on the second derivative of the potential, in agreement with the dS conjecture considerations, although they also apply to AdS vacua. In this simple one-modulus case one can show that dS minima are forbidden.

In section 5 we consider the more speculative possibility that the moduli themselves have masses subject to the same constraints as the WGC states which obey the conditions. 
This gives rise to constraints involving 3-d and 4-th derivatives of the scalar potential, analogous to those discussed in ref. [15] but with an absolute value taken. Some particle physics and cosmology implications from that kind of constraint were described in that reference. However the presence of the absolute value changes some of the consequences. In particular, the condition in the present case disappears when gravity decouples and no restriction on scalar field ranges appear in the field theory in the infrared.

The idea underlying our pair-production proposal is not to put it forward as an alternative to long range one-particle exchange arguments. Our point of view is rather that the hypothesis of gravity being the weakest force could be tested in different particle configurations and kinematic limits. Each of them may be optimal to test a particular property of the WGC ideas. The Pair-Production proposal is an S-matrix criterion and is optimal to test the WGC when scalar interactions are involved. The general idea may, in principle, be applied in any number of dimensions. Nevertheless, in this work we restrict our computations and arguments to $d=4$. The Pair-Production criteria may actually turn out to be closely related to black-hole decay and the standard WGC. Whereas usual WGC arguments based on stability of extremal black-holes are purely kinematical, our condition may perhaps point to an additional dynamical condition.

The structure of this paper is as follows. In the next section we study the PPWGC for the case of $\mathrm{U}(1)$ interactions. We first compute the production rate at threshold of both charged scalar and fermion pairs from photons and gravitons. We show how insisting on the graviton rate being smaller than the photon rate reproduces the usual U(1) WGC constraint. We also generalise the constraint to the multiple $\mathrm{U}(1)$ case. In section 3 we study the PPWGC for scalars, and compute the production rate of a pair of heavy scalars from the collision of two moduli. Insisting that this rate is larger than the rate from graviton production we obtain the Scalar WGC constraint. We apply it to the case of complex and real scalars and study the structure of the massive states which saturate the bounds. Several examples are presented and consistency with known $N=2$ BPS results is shown. Section 4 study possible connections with the dS conjecture and section 5 briefly discusses the case of the Strong or generalized Scalar WGC's in which the masses of the moduli are assumed to obey the same constraints as the massive WGC states discussed in the previous sections. Some final comments are presented in section 6 .

\section{The PPWGC for $\mathrm{U}(1)$ interactions}

\subsection{A single $\mathrm{U}(1)$}

In this section we study the case of a single $\mathrm{U}(1)$ with pair production of charged scalars and fermions.

Let us start with the production of scalars. The relevant diagrams are shown in figure 1. For the photon production we are not including a diagram in which the two photons go to a graviton which then produce two scalars. The reason is that it is purely gravitational and hence should not be included if our aim is to compare a purely electro-magnetic production with a purely gravitational production. Thus for the photon production we are taking the 


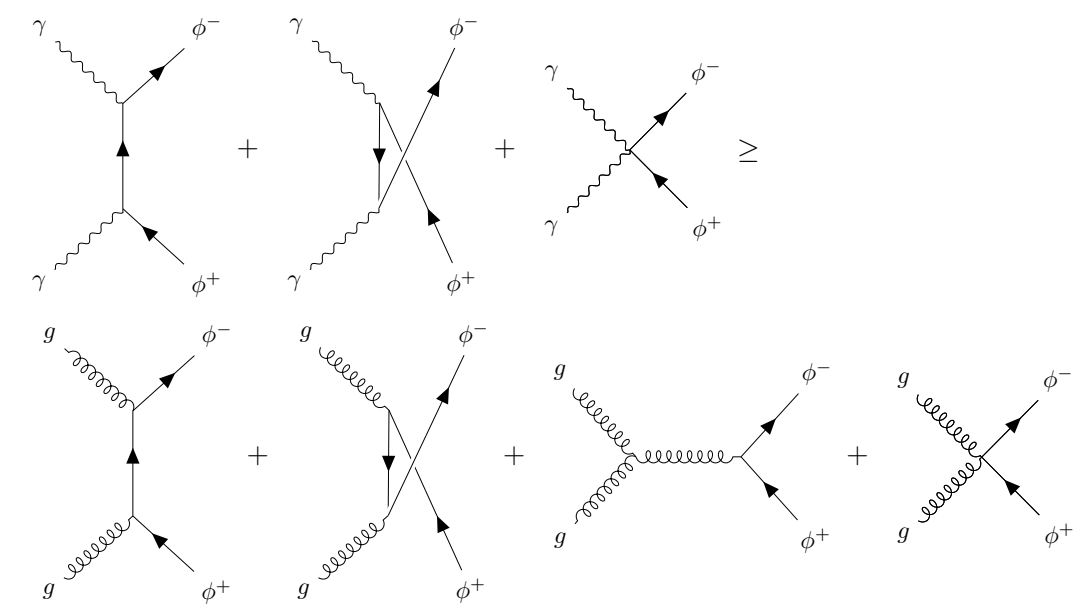

Figure 1. The relevant tree level diagrams for the pair production of charged scalars in SQED and linearized Einstein gravity. We assign the letter $A$ to the diagrams with photons and the letter $\mathrm{C}$ to the production via gravitons.

$M_{p} \rightarrow \infty$ limit. The cross sections for photon and graviton pair production in the CM are written as

$$
\left(\frac{d \sigma}{d t}\right)_{\mathrm{CM}}^{\mathrm{SQED}}=\frac{|A|^{2}}{32 \pi s^{2}} ; \quad\left(\frac{d \sigma}{d t}\right)_{\mathrm{CM}}^{\operatorname{Grav} \phi^{*}}=\frac{|C|^{2}}{32 \pi s^{2}} .
$$

At threshold the four-momentum of the final particles is $p=(m, \overrightarrow{0})$ and the cross section vanishes. We are not interested in comparing the cross section at threshold, but in the threshold limit, where the particles in the produced pair have infinitesimal but non-zero momenta. Thus, what we will compare is the differential cross section with respect to $t$. In the threshold limit the Mandelstman variables are given by: $t=u=-m^{2}$ and $s=4 m^{2}$. Using the helicity formalism, we will see that at threshold only the amplitudes where the initial photons or gravitons have opposite helicities contribute. Both amplitudes have the structure

$$
|M|^{2}=2\left|M_{++}\right|^{2}+2\left|M_{+-}\right|^{2} .
$$

For the photon production amplitude one obtains

$$
A_{+-}=\frac{2 e^{2}\left(m^{4}-u t\right)}{\left(t-m^{2}\right)\left(u-m^{2}\right)} ; \quad A_{++}=-\frac{2 e^{2} m^{2} s}{\left(t-m^{2}\right)\left(u-m^{2}\right)} .
$$

The computation of the graviton production is non-trivial. Fortunately, the rate may be obtained by crossing from the graviton Compton scattering computed in [14]. Interestingly, one finds that the gravitational amplitudes for the Compton scattering of a spin $S$ particle with gravitons are given by the product of the electromagnetic Compton scalar amplitude times the electromagnetic amplitude for a spin $S$ particle [29-31]: ${ }^{1}$

$$
\left|C_{+-}\right|^{2}=\left|C_{-+}\right|^{2}=F^{2}\left|A_{+-}\right|^{4} ; \quad\left|C_{++}\right|^{2}=\left|C_{--}\right|^{2}=F^{2}\left|A_{++}\right|^{4},
$$

\footnotetext{
${ }^{1}$ This is an avatar of the (gravity) $=(\text { gauge })^{2}$ property of scattering amplitudes, see e.g. [32, 33] and references therein.
} 


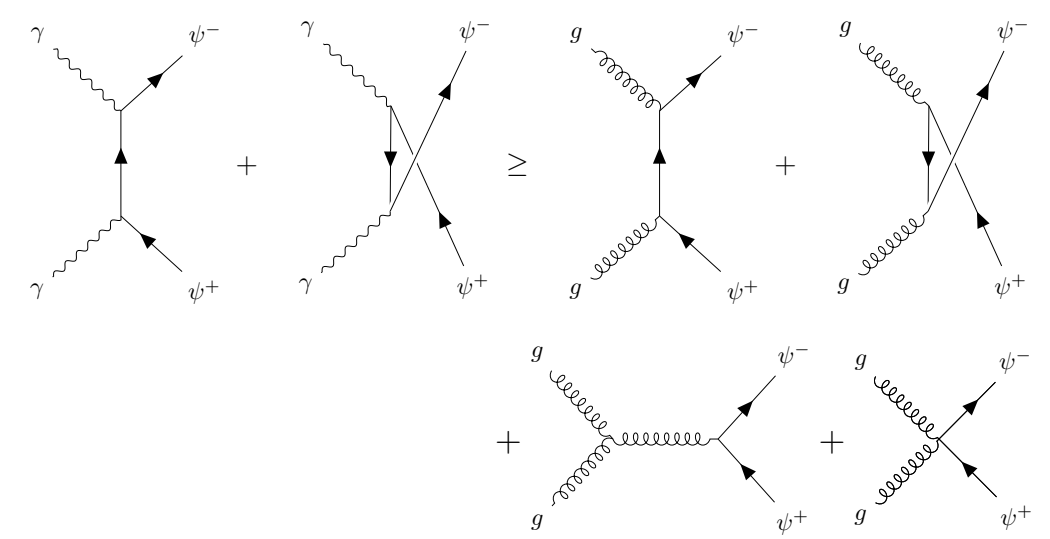

Figure 2. Tree level diagrams contributing to the pair production in QED and linearized Einstein gravity. We assign the letter $B$ to the diagrams with photons and the letter D to those with gravitons.

where

$$
F=\frac{1}{4 M_{p}^{2} e^{4}} \frac{\left(t-m^{2}\right)\left(u-m^{2}\right)}{s} .
$$

At threshold one has $s=4 m^{2}, t=u=-m^{2}$ and one obtains

$$
\left|A_{+-}\right|^{2} \longrightarrow \quad\left|A_{++}\right|^{2} \longrightarrow 4 e^{4} \quad \text { and } \quad\left|C_{+-}\right|^{2} \longrightarrow 0, \quad\left|C_{++}\right|^{2} \longrightarrow \frac{m^{4}}{M_{p}^{4}}
$$

The PPWGC then gives us:

$$
|A|^{2} \geq|C|^{2} \longrightarrow \sqrt{2} e \geq \frac{m}{M_{p}}
$$

in agreement with the standard constraint of the WGC for a single $\mathrm{U}(1)$. The factor $\sqrt{2}$ is important since it is precisely the factor that appears for extremal Reissner-Nordstrom black-holes.

For completeness, let us consider now the spin $1 / 2$ fermion production, although in the rest of the paper we will concentrate on the production of scalars. The relevant diagrams are shown in figure 2. We sum over spins in the final state in both rates. Denoting $B$ and $D$ the photon and graviton amplitudes respectively one finds

$$
\begin{gathered}
\left|B_{+-}\right|^{2}=\frac{4 e^{4}\left(m^{4}-u t\right)\left[2\left(m^{4}-u t\right)+s^{2}\right]}{\left(t-m^{2}\right)^{2}\left(u-m^{2}\right)^{2}} \longrightarrow 0 \\
\left|B_{++}\right|^{2}=\frac{4 m^{2} e^{4} s^{2}\left(2 m^{2}-s\right)}{\left(t-m^{2}\right)^{2}\left(u-m^{2}\right)^{2}} \longrightarrow 8 e^{4}, \\
\left|D_{++}\right|^{2}+\left|D_{+-}\right|^{2}=F^{2}\left(\left|A_{++}\right|^{2}\left|B_{++}\right|^{2}+\left|A_{+-}\right|^{2}\left|B_{+-}\right|^{2}\right) \longrightarrow \frac{2 m^{4}}{M_{p}^{4}},
\end{gathered}
$$

where we have already indicated the value at threshold. Then PPWGC also gives us

$$
|B|^{2} \geq|D|^{2} \longrightarrow \sqrt{2} e \geq \frac{m}{M_{p}}
$$


as expected. Thus we see that, imposing that the pair production rate of charged particles at threshold to be larger than the rate for the production from gravitons, we obtain the same constraint as the standard U(1) WGC. A proportionality between charges and masses in the rate was to be expected. But, as we have shown, the fact that all precise factors match is non-trivial. It is also a test that the pair production at threshold of an extremal state has equal probability either from photons or gravitons. Using crossing symmetry, this also implies that the annihilation rate of extremal particles at rest into photons and gravitons is the same.

\subsection{Multiple U(1)'s}

Consider now $N \mathrm{U}(1)$ gauge bosons with a diagonal and canonical kinetic term. We should now insist that a particle with mass $m$ and charge vector $\vec{Q}=\left(Q_{1}, \ldots, Q_{N}\right)$ must exist so that its pair production by photons is equal or bigger than its pair production by gravitons. The calculation of the rates in the previous section is trivially extended for multiple $\mathrm{U}(1)$ and gives:

$$
\left(Q_{1}^{4}+Q_{1}^{2} Q_{2}^{2}+Q_{2}^{2} Q_{1}^{2}+\ldots+Q_{N}^{4}\right)=\left(\vec{Q}^{2}\right)^{2} \geq \frac{m^{4}}{4 M_{p}^{4}} .
$$

The general statement of the PPWGC applied to this case would say that for every rational direction in the charge lattice there is a particle of mass $m$ whose photon production rate at threshold is larger than its graviton production rate. Note that the produced objects must be stable or metastable particles, for the Feynman graph computation to make sense.

To shorten notation we can say that a charged state is superproduced if the rate to produce a pair of such particles at threshold is larger or equal to the rate to produce that pair from gravitons. Then the above conjecture may be restated as:

The Pair Production Weak Gravity Conjecture (PPWGC) for Photons. For any rational direction in the charge lattice $\vec{Q}$ there is a (meta)stable particle which is superproduced.

Here a rational direction is a ray in the charge lattice, passing through both the origin and $\vec{Q}$. We chose to impose the PPWGC for every rational direction in the charge lattice. A motivation for this choice is that the superproduced particle acts also as a standard WGC state to which extremal black-holes can decay. Note that the PPWGC so defined includes the WGC but it is stronger. Let us review the latter to ilustrate this point. As stated e.g. in [10] the WGC reads:

The Weak Gravity Conjecture (WGC). For every rational direction in the charge lattice there is a superextremal multiparticle state.

A superextremal state is one whose $\vec{Z}=\vec{Q} / m$ is either outside or on the boundary of the black-hole region. For the theory we are considering the black-hole region is simply given by $M_{\mathrm{BH}} \leq \sqrt{2}|\vec{Q}|_{\mathrm{BH}} M_{p}$. Therefore, from (2.12) we can see that, in this context with no scalar fields, superproduced is equivalent to superextremal. Figure 3 (an adaptation of a figure in [28]) illustrates the relation between the statements of the WGC and PPWGC 


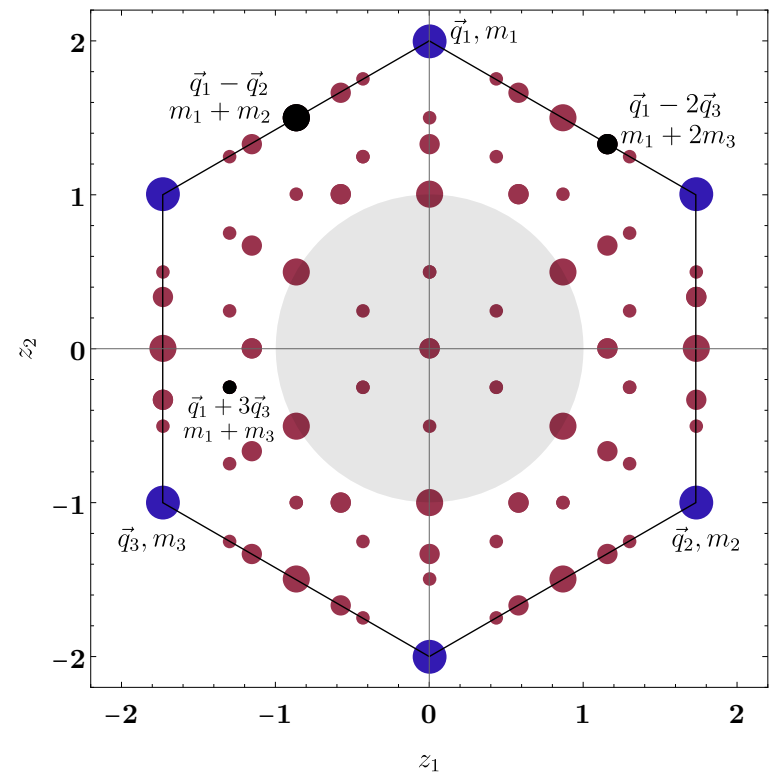

Figure 3. Multi-particle states of a $\mathrm{U}(1)^{2}$ with three fundamental particles and their corresponding antiparticles in the spectra. These six particles are displayed with blue dots. The maroon dots are multi-particle states formed from them. The more particles a state has, the smaller the size of the dot representing it. For illustrative purposes we have written the charge and the mass of three randomly chosen states, which appear in black in the figure. We use lower-case letters $\vec{z}$ and $\vec{q}$ to refer to the charges of the single-particle states. The black-hole region is represented by a grey circle. We have chosen $m_{1}=m_{2}=m_{3}=1$ and $\overrightarrow{q_{1}}=(0,2), \overrightarrow{q_{2}}=(\sqrt{3},-1), \overrightarrow{q_{1}}=(-\sqrt{3},-1)$. Therefore, the convex hull encloses the black-hole region in our example.

with the well-known Convex Hull Condition (CHC). In this figure we considered a $\mathrm{U}(1)^{2}$ with three fundamental (not composite) particles and their corresponding antiparticles in the spectra. These six particles are displayed with blue dots. The maroon dots are multiparticle states formed from them. For illustrative purposes we have written the charge and the mass of three randomly chosen states, which appear in black in the figure. The more particles a state has, the smaller the size of the dot representing it. One can see that the multi-particle states populate the convex hull of the fundamental particles in $\vec{Z}$ space. The black-hole region is represented by a grey circle in the figure. If for every rational direction there is a superextremal state, then the convex hull encloses the black-hole region.

Notice that, unlike the PPWGC, black-hole arguments do not care whether the state is single or multi-particle. For us though it is not enough to have a superproduced multiparticle state to ensure that gravity is the weakest force, we actually need a pair of particles, possibly metastable. Thus, the PPWGC is similar to the standard WGC, but the constraint it puts on the spectra is actually stronger than the CHC. The key point is that in the PPWGC approach we are producing actual particles.

It has been noted that examples from gravity and string theory suggest that a stronger version of the WGC for $\mathrm{U}(1)^{N}$ is required in order to be preserved under dimensional reduction. Two closely-related strong forms are particularly well motivated: the Sublattice WGC (sLWGC) [7] and the Tower WGC (TWGC) [9]. Both require the existence of an 
infinite number of superextremal particles along each rational direction in charge space. In this sense the PPWGC is very similar to the stronger versions of the WGC. Instead of imposing that a superproduced particle must exist for every rational direction we could have, in fact, directly imposed the tower or sublattice versions:

The Tower Pair Production WGC (TPP-WGC). At any point $\vec{q}$ of the charge lattice there exists a positive integer $n$ such that there is a superproduced particle of charge $n \vec{q}$.

The Sublattice Pair Production WGC (sPP-WGC). There exists a positive integer $n$ such that for any site $\vec{q}$ in the charge lattice there is a superproduced particle of charged $n \vec{q}$.

Notice that the main difference between Tower and Sublattice conjectures is that in the latter the integer $n$ is universal. It is interesting that the PPWGC is sensitive to whether the WGC state is a single or a multi-particle state.

\section{Pair production from scalars and the Scalar Weak Gravity Conjecture}

Once we have seen how the PPWGC criterium encompasses the WGC conjecture and its extensions, we will now show how its application to production from scalars leads to interesting novel results. The original formulation of the WGC rested on energy and charge conservation in extremal black-hole decay. The absence of proper scalar charges makes a parallel reasoning difficult. In this section we apply the principle of the Pair-Production WGC, to theories with scalar fields. The particular inequality which is obtained from the general formula eq. (1.1) will depend on the geometry of the scalar manifold we are studying, so we will consider different possibilities.

We will take in all our examples and constraints the case of massless scalars, like moduli in string theory. In theories with supersymmetry they may remain massless over all moduli space. So in some of the examples the massless scalars may be considered as a bosonic subsector of a SUSY theory. Still, the principle of gravity being the weakest form seems unrelated to supersymmetry, and the idea would be that the constraints obtained should also apply to non-SUSY theories in which for some reason the scalars remain much lighter than the Planck scale.

Let us start with the simple case of a massless complex scalar field $T$ and a complex heavy scalar field $H$ with a mass $m^{2}\left(T, T^{*}\right)$. The relevant part of the action has a structure

$$
\mathcal{L}_{\mathrm{T}}=\partial_{\mu} H \partial^{\mu} \bar{H}+\partial_{\mu} T \partial^{\mu} \bar{T}-m^{2}\left(T, T^{*}\right)|H|^{2},
$$

with a moduli dependent mass term for the heavy scalar $H$. It is always possible to expand $m^{2}$ at a generic point in moduli space up to second order in the fields, and write the result in terms of either real or complex components. In terms of the complex variables:

$$
m^{2} \simeq m_{0}^{2}+\left(\partial_{T} m^{2}\right) T+\left(\partial_{\bar{T}} m^{2}\right) \bar{T}+\left(\partial_{\bar{T}} \partial_{T} m^{2}\right)|T|^{2}+\frac{1}{2} \partial_{T}^{2} m T^{2}+\frac{1}{2} \partial_{\bar{T}}^{2} m \bar{T}^{2} \ldots
$$

Following the PPWGC, we ought to consider the pair production of the field $H$ and compare it with the production from gravitons at threshold. The relevant diagrams for the 


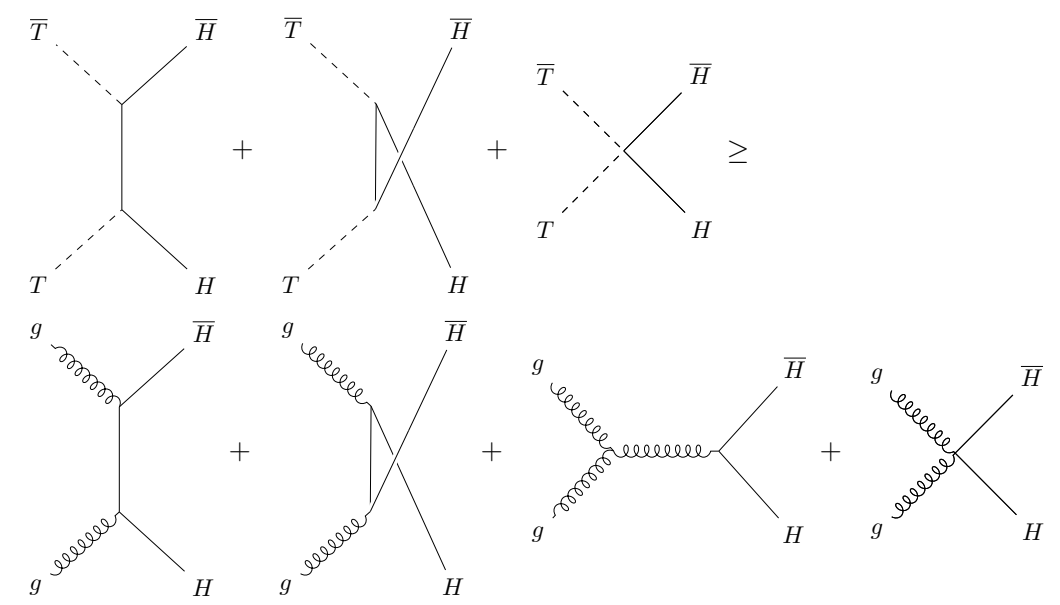

Figure 4. Tree level diagrams contributing to the pair production in the scalar theory and linearized Einstein gravity. We assign the letter $N$ to the diagrams with moduli.

process $T \bar{T} \rightarrow H \bar{H}$ are shown in figure 4. Again, in the production from scalars we are not including the annihilation of two scalars into a graviton, with the latter producing heavy pairs, since it is purely gravitational and does not involve scalar couplings. Notice also that the last two terms in the $m^{2}$ expansion will not contribute to the four point function that we are interested in, where the initial particles are a pair $T, \bar{T}$. From the expansion we extract the trilinear $\Delta T|H|^{2}+$ h.c. and the quartic $\lambda|T|^{2}|H|^{2}$ couplings:

$$
\Delta=\partial_{T} m^{2}, \bar{\Delta}=\partial_{\bar{T}} m^{2}, \quad \lambda=\partial_{T} \partial_{\bar{T}} m^{2} .
$$

The amplitude has the form:

$$
N=-|\Delta|^{2}\left[\frac{1}{t-m^{2}}+\frac{1}{u-m^{2}}\right]-\lambda
$$

The gravitational diagrams are the same as in section 2. At threshold one has $t=u=-m^{2}$, and the condition reads

$$
\left|\frac{|\Delta|^{2}}{m^{2}}-\lambda\right|^{2} \geq \frac{m^{4}}{M_{p}^{4}}
$$

In terms of mass derivatives one obtains

$$
\left|\left(\partial_{T} m^{2}\right)\left(\partial_{\bar{T}} m^{2}\right)-m^{2} \partial_{T} \partial_{\bar{T}} m^{2}\right| \geq \frac{m^{4}}{M_{p}^{2}} .
$$

For $n$ complex moduli $T_{i}, i-1, \ldots, n$ parameterising a hermitian manifold with a metric $g_{i \bar{j}}$ this is generalised to

$$
\frac{g^{i \bar{j}}}{n}\left|\left(\partial_{i} m^{2}\right)\left(\partial_{\bar{j}} m^{2}\right)-m^{2}\left(\partial_{i} \partial_{\bar{j}} m^{2}\right)\right| \geq \frac{m^{4}}{M_{p}^{2}} .
$$

This is the general form of the scalar WGC for $\mathrm{n}$ complex moduli. Notice that, as expected, this expression is invariant under holomorphic coordinate transformations. We could replace the partial derivatives with covariant derivatives, however, nothing would change 


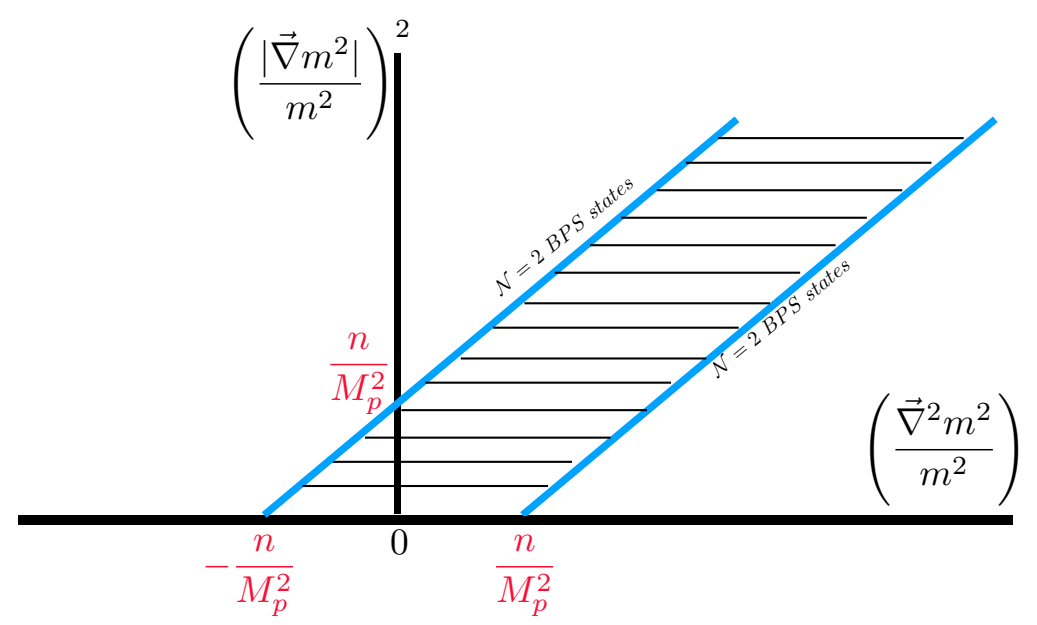

Figure 5. The scalar WGC for $n$ active moduli coupling to massive WGC scalars. The barred region correspond to points in moduli space in which gravity is too strong. This region disappears as $M_{p} \rightarrow \infty . N=2$ BPS states lie on top of the blue boundary lines.

since the mixed index components of the connection vanish in a hermitian manifold. In order to compare the contribution of the moduli to graviton production an averaging factor $1 / n$ is included. In other words, the contribution of all moduli should be compared with $n$-times the production rate from gravitons in order to have a fair comparison. Such an averaging was not needed in the case of production from photons in an $\mathrm{U}(1)^{n}$ theory with canonical kinetic basis because for any given charged particle one can always find a basis in which it couples to a single $\mathrm{U}(1)$. Note that ' $n$ ' here refers only to active moduli i.e. the subset of the moduli in the theory which couple to a particular massive state. A graphical interpretation of this constraint is given in figure 5 . The region between the two parallel lines is forbidden, but it disappears as $M_{p} \rightarrow \infty$, as is expected from a swampland condition. Points in field space saturating the bound lie on top of the blue lines marking the boundaries.

One can then state

The Pair Production Scalar Weak Gravity Conjecture (PPSWGC). Given any set of moduli scalars, there must be a massive particle $H$ with mass $m$ coupled to them such that their average production rate at threshold from moduli is larger than the corresponding rate from gravitons.

\subsection{Examples}

\subsubsection{Complex scalar in $N=1$ supergravity}

Consider first the case of $N=1$ supergravity with a metric $g_{i \bar{j}}=K_{i \bar{j}}$, with $K\left(T, T^{*}\right)$ the Kahler potential. Without loss of generality let us define the real function $F\left(T_{i}, T_{i}^{*}\right)$ by $m^{2}=M_{p}^{2} e^{F}$, and take n complex $T_{i}$ fields dimensionless. One can check that eq. (3.7) may be rewritten in the simple form

$$
g^{i \bar{j}}\left|F_{i \bar{j}}\right| \geq n .
$$


Note that, due to the absolute value, there is a symmetry under $F \leftrightarrow-F$. This tells us that, if there is a particle with mass $m$ verifying the bound, a particle with mass $m^{\prime}=M_{p}^{2} / m$ would also obey it. In the specific models below this symmetry would correspond to a duality symmetry. Note also that in a $N=1$ supergravity theory with spontaneously broken SUSY, the gravitino mass may be written as $m_{3 / 2}^{2}=e^{G}$, with $G$ the full Kahler potential. With this structure such a mass automatically saturates the bound, which would apply rather to the scalar s-Goldstino, since the massive states in our derivation are scalars.

Let us consider the simple case in which the moduli have a no-scale metric, i.e., $g_{i, \bar{j}}=\delta_{i, \bar{j}} /\left(T_{i}+T_{i}^{*}\right)^{2}$. These appear for example in $N=1$ toroidal/orbifold compactifications down to $4 \mathrm{D}$ in string theory (see e.g. [34]). The conjecture requires now the existence of scalar fields, with mass $m^{2}\left(T_{i}, T_{i}^{*}\right)$, coupled to the moduli. The constraint is in this example

$$
\delta^{i \bar{j}}\left(T_{i}+T_{i}^{*}\right)^{2}\left|F_{i \bar{j}}\right| \geq n .
$$

Let us study the case in which the inequality saturates. One finds solutions

$$
F=f\left(T_{i}\right)+f^{*}\left(T_{i}^{*}\right)+\sum_{i} \eta_{i} \log \left(T_{i}+T_{i}^{*}\right)
$$

with $f\left(T_{i}\right)$ an arbitrary holomorphic function. Here $\eta_{i}$ takes all possible choices $\eta_{i}= \pm 1$. In this case our PPWGC saturating states would have a mass

$$
m_{a}^{2}=m_{0}^{2}\left|e^{f\left(T_{i}\right)}\right|^{2} t_{1}^{ \pm 1} t_{2}^{ \pm 1} \ldots t_{n}^{ \pm 1}
$$

with $f\left(T_{i}\right)$ an arbitrary holomorphic function and $t_{i}=2 \operatorname{Re} T_{i}$. The index $a=1,2, \ldots 2^{n}$. The large modulus behaviour depends on the form of the holomorphic functions $f\left(T_{i}\right)$. In the case of constant f's, going to a canonical frame with $t=e^{\sigma}$ there are states which become exponentially light in the limits $\sigma \rightarrow \pm \infty$. This behaviour would be in agreement with the expectations of the swampland distance conjecture. Also for each saturating state there is another dual state with inverse mass, as pointed out above. We will see below that certain classes of BPS states in known $N=2$ supergravity theories from string theory are consistent with this structure.

For more general CY the metric of the Kahler moduli (in. e.g. Type IIA string theory) has the behaviour at large moduli

$$
K_{i \bar{j}} \simeq \frac{d_{i}}{\left(T_{i}+T_{\bar{i}}\right)^{2}}
$$

where the $d_{i}$ are integers characteristic of each singular limit [17-19]. From eq. (3.8) one can compute the asymptotic behaviour of the particles which saturate our bound. One now finds

$$
m_{a}^{2}=m_{0}^{2}\left|e^{f\left(T_{i}\right)}\right|^{2}\left(t_{1}^{ \pm d_{1}} \ldots t_{n}^{ \pm d_{n}}\right) .
$$

This behaviour, corresponds e.g. to the asymptotic behaviour found in $[17,18]$, showing the large moduli regime of BPS states in Type IIB CY compactifications. It would be interesting to go through examples in e.g. [18-23] and check the agreement with the constraint. 


\begin{tabular}{|c|c|c|c|c|}
\hline & D0 & D2 & D4 & D6 \\
\hline $\operatorname{Mass}^{2}(\mathrm{CY})$ & $e^{K_{K}}$ & $e^{K_{K}}\left|T_{a}\right|^{2}$ & $e^{K_{k}}\left|\frac{1}{2} \sum_{b, c} \kappa_{a b c} T^{b} T^{c}\right|^{2}$ & $e^{K_{k}\left|\frac{1}{6} \sum_{a, b, c} \kappa_{a b c} T^{a} T^{b} T^{c}\right|^{2}}$ \\
\hline $\operatorname{Mass}^{2}\left(Z_{2} \times Z_{2}\right)$ & $\frac{1}{t^{1} t^{2} t^{3}}$. & $\frac{t^{i}}{t^{j} t^{k}}$ & $\frac{t^{j} t^{k}}{t^{i}}$ & $t^{1} t^{2} t^{3}$ \\
\hline
\end{tabular}

Table 1. Masses of the different particles obtained by wrapping one kind of Type IIA D $p$-brane around a given even cycle on a general Calabi-Yau threefold and for the $Z_{2} \times Z_{2}$ orbifold example from ref. [20]. Masses are in units of $8 \pi M_{p}^{2}$.

The solutions in (3.11) allow for alternative behavior depending on the particular holomorphic functions $f\left(T_{i}\right)$, the arbitrariness is substantial. For example, one may chose all $\eta_{i}=-1$ and $f=-\log \left(\Pi_{i} \eta\left(T_{i}\right)^{2}\right)$, with $\eta$ the Dedekind function. With such a choice the mass is $\operatorname{SL}(2, \mathbf{Z})^{n}$ invariant. This kind of structure appears in the class of duality invariant non-perturbative potentials considered in [24] and references therein. At large moduli the Dedekind function has an exponential behaviour $\eta \sim e^{-(\pi / 12) t}$, so that there could be saturating solutions with a behaviour $m^{2} \sim 1 /\left(\Pi_{i} t_{i} e^{-\pi / 3 t_{i}}\right)$, exponentially growing at large $t_{i}$. This class of solutions would not have the asymptotic behaviour of the distance conjecture, as explained in [24]. Note in this respect that such exponential of exponential behaviour at large moduli appears also for the states called of Type II in [18, 19] for Type II CY compactifications.

\subsubsection{Examples from BPS states in $N=2$ supergravity}

We would like now to show that examples of BPS states in $N=2$ supergravity theories from string theory saturate our bound. We will consider for illustration a class of BPS states which appear in Type IIA CY compactifications from $D p$-branes wrapping even cycles. These (and their IIB mirrors) have been discussed in [17-22] to provide string theory tests of the swampland distance conjecture. We follow here [20]. The relevant masses are summarized in the table. Here $K_{K}$ is the Kahler potential of the Kahler moduli $T_{a}=t_{a}+i \eta_{a}, a=1, \ldots, h_{11}$, and $\kappa_{a b c}$ are the triple intersection numbers in the CY. The masses of the BPS states may be written as $m_{r}^{2}=8 \pi e^{G_{r}}$, where

$$
G_{r}=\log \left|W_{r}\right|^{2}+K_{K},
$$

and $\left|W_{r}\right|^{2}$ is given by the different superpotential factors in the table. With this form one obtains the constraint is

$$
g^{i \bar{j}}\left|\left(G_{r}\right)_{i \bar{j}}\right|=h_{11}
$$

which holds, since $\left(G_{r}\right)_{i \bar{j}}=\left(K_{K}\right)_{i \bar{j}}=g_{i \bar{j}}$. More explicitly, for the case of the $Z_{2} \times Z_{2}$ toroidal example considered in [20] there are three Kahler moduli $T_{i}$ and 8 BPS states corresponding to D0,D2,D4 and D6 wrapping even cycles. Their masses are $m_{r}^{2}=8 \pi\left(t_{1}^{ \pm 1} t_{2}^{ \pm 1} t_{3}^{ \pm 1}\right)$, with $t_{i}=2 \operatorname{Re} T_{i}$, as shown in table 1 . Note that these masses agree with the result we showed in eq. (3.11) (for $f$ constant) which do saturate our bound.

Note that, in agreement with the duality symmetry $F \leftrightarrow-F$, for each BPS example in the table with mass $m$, there is another one with mass $1 / m$. From the D-brane perspective, 
a duality with respect to the six compact dimensions transforms $D_{0} \leftrightarrow D_{6}$ and $D_{2} \leftrightarrow D_{4}$. This is also an electric-magnetic duality since the states have also inverse charge under magnetic U(1)'s. In figure 5 particles coming from $D 0, D 2$ are points within the rightmost blue line, whereas those coming from $D 4, D 6$ are inside the blue line on the left.

The fact that for these $N=2$ BPS states our condition is saturated is not surprising due to the following fact. In $N=2$ supergravity the central charge $Z$ satisfies the algebraic equation $[11,13,35]$

$$
g^{i \bar{j}}\left(D_{i} \bar{D}_{\bar{j}}|Z|^{2}-D_{i} Z \bar{D} \bar{j} \bar{Z}\right)=n_{V}|Z|^{2}
$$

where $n_{V}$ counts the number of vector multiplets. This condition should be verified by the central charge of any BPS state. The above algebraic expression may be derived from the Special Kahler Geometry identities (see e.g. [36])

$$
D_{\bar{i}} Z=0 ; \quad D_{i} \bar{D}_{\bar{j}} Z=g_{i \bar{j}} Z .
$$

Identifying $Z$ with the ADM mass $m$ suggests to write

$$
m^{2} g^{i \bar{j}} \partial_{i} \partial_{\bar{j}} m^{2}-\left(\partial_{i} m^{2}\right)\left(\partial^{i} m^{2}\right)=n_{V} m^{4}
$$

This equation is consistent with our equation (3.7) above and masses saturating it would lie at the boundary blue lines in figure 5 .

\subsubsection{The case of $\mathbf{n}$ real scalar fields}

Let us consider now the case of $n$ real scalar fields $t_{i}$ with diagonal kinetic terms. One can obtain the trilinear and quartic couplings from the general expansion

$$
m^{2}\left(t_{i}\right) \simeq m_{0}^{2}+\left(\partial_{i} m^{2}\right) t_{i}+\frac{1}{2}\left(\partial_{i}^{2} m^{2}\right) t_{i}^{2}+\ldots
$$

Consider first the case of $n$ real scalars with diagonal no-scale kinetic metrics $g_{i i}=1 / t_{i}^{2}$. From the pair production constraint we now obtain

$$
\sum_{i} g^{i i}\left|\left(\partial_{i} m^{2}\right)^{2}-m^{2}\left(\partial_{i}^{2} m^{2}\right)\right| \geq n \frac{m^{4}}{M_{p}^{2}}
$$

Writting $m^{2}=e^{F}$, when the inequality is saturated one obtains

$$
\sum_{i}\left(t_{i}^{2}\right)\left|\partial_{i}^{2} F\right|=n
$$

with solutions

$$
m^{2}\left(t_{i}\right)=m_{0}^{2}\left(t_{i}^{ \pm 1} \ldots t_{n}^{ \pm 1}\right) e^{\sum_{i} b_{i} t_{i}} .
$$

Here $m_{0}$ and $b_{i}$ are real integration constants. Note that now, unlike the SUSY case above, the existence of a scalar moduli space is not expected, and the interpretation of the massive states saturating the bounds is not obvious. Still, it is interesting to explore for comparison what is the form of the saturating masses in this case. We have two classes of solutions. For $b_{i}=0$ one obtains saturating states very similar to the complex no-scale metric example in 
eq. (3.11), which is the kind of behavior of BPS, KK and winding states in string theory. As in the previous examples, this is in itself remarkable, since it means that the scalar PPWGC condition for massless particles with scale invariant metrics must come along with a massive spectrum behaving like winding and momenta, i.e., string theory. Note also that here the presence of both winding and momenta (and hence duality) is a consequence of the invariance under $F \rightarrow-F$ of the rates. Thus having rates and no amplitudes in comparing the interactions is at the root of the built-in duality of the massive spectrum. Going to a canonical frame with $t=e^{\sigma}$ the behavior at large $\sigma$ is exponential, consistent with the distance swampland conjecture.

For $b_{i} \neq 0$ there are additional saturating solutions. They have an additional exponential factor in $t$, which means exponential of exponentials once one goes to a canonical frame. This is analogous to the result above for complex moduli.

We may alternatively consider a canonical metric for the scalar fields. It is easy to see that in this case one obtains saturating solutions of the form

$$
m^{2}\left(t_{i}\right)=m_{0}^{2}\left(t_{1}^{d\left(t_{1}\right)^{ \pm}} \ldots t_{n}^{d\left(t_{n}\right)^{ \pm}}\right), \quad d\left(t_{i}\right)^{ \pm} \equiv c_{ \pm}^{i} \pm 1 / 2 \log (t),
$$

where we have defined $t_{i}=e^{\phi_{i}}$, with $\phi$ the canonical fields and $c_{ \pm}^{i}$ and $m_{0}^{2}$ are real constants. The structure has also a form proportional to powers $t^{d(t)}$, reminiscent of the of the examples discussed above, but now with a slowly varying exponent $d(t)$. Under a duality transformation $t_{i} \rightarrow 1 / t_{i}$ one obtains a new solution exchanging $c_{+}^{i} \rightarrow-c_{+}^{i}$, $d\left(t_{i}\right)_{+} \rightarrow-d\left(t_{i}\right)_{+}$. So again for each solution of mass $m$ there is another solution with mass $1 / \mathrm{m}$. Note however that going to a canonical frame shows that the solutions have a Gaussian behavior. In fact such Gaussian solutions would also appear for the case of complex scalar fields with a canonical, instead of no-scale metrics.

\subsubsection{Previous formulations of the SWGC}

There have been previous formulations of scalar weak gravity conjectures in the literature. Palti was the first in making the proposal [11] that a theory with moduli $t^{i}$ should have a state $H$ with mass $m$ obeying the bound

$$
g^{i j}\left(\partial_{t^{i}} m\right)\left(\partial_{t^{j}} m\right)>\frac{m^{2}}{M_{p}^{2}} .
$$

This has the simple interpretation of imposing that a trilinear $t^{i}|H|^{2}$ coupling squared is stronger than the gravitational coupling. In that paper it was noted that this inequality cannot be directly deduced from bound states arguments (or from the RFC) since both the scalars and gravity act attractively. It was also noticed that, at large field, this expression is consistent with the swampland distance conjecture. Palti also proposed (see footnote in [11]) the inequality

$$
\frac{1}{2} g^{i j} \nabla_{t^{i}} \nabla_{t^{j}} m^{2}-g^{i j}\left(\partial_{t^{i}} m\right)\left(\partial_{t^{j}} m\right) \geq n \frac{m^{2}}{M_{p}^{2}},
$$

with $n$ the number of real scalars coupling to the WGC state of mass $m$. The motivation for this inequality mainly came from the Special Geometry identities in $N=2$ supergravity 
mentioned above. Note that it is analogous to our constraint except for the fact that we have an additional absolute value taken in the left (and there is some numerical factor).

In ref. [15] it was proposed a slightly different version of a scalar WGC for a real scalar with canonical metric given by

$$
2\left(\partial_{\phi} m^{2}\right)^{2}-m^{2} \partial_{\phi}^{2} m^{2} \geq \frac{m^{4}}{M_{p}^{2}} .
$$

The motivation was to modify the original scalar WGC of eq. (3.24) to include quartic scalar interactions. A further motivation was the intriguing structure of its saturating solutions. Indeed the above equation may be rephrased as

$$
\rho^{\prime \prime}=\frac{\rho}{M_{p}^{2}}, \quad \rho \equiv \frac{1}{m^{2}},
$$

and the saturating solutions have the form

$$
m^{2}=\frac{1}{a e^{\phi}+b e^{-\phi}} .
$$

At both limits $\phi \rightarrow \pm \infty$ the behaviour is consistent with the swampland distance conjecture. Furthermore, defining $t=e^{\phi}$, there is built-in duality under the exchange $t \leftrightarrow 1 / t$. Thus the saturating solutions have the structure of KK and winding momenta, implying the existence of an underlying theory with extended objects. This is in fact the kind of structure that we have found in the present article, although the precise form of the constraint is not the same.

For a single real massless modulus and a massive state of mass $m$, the scalar PPWGC gives rather the constraint eq. (3.20)

$$
\left|\left(\partial_{\phi} m^{2}\right)^{2}-m^{2}\left(\partial_{\phi}^{2} m^{2}\right)\right| \geq \frac{m^{4}}{M_{p}^{2}}
$$

Comparing with (3.26) we see there is a factor 2 missing in the first term and the absolute value taken on the left. It is the factor 2 which makes the solutions in (3.28) different from those of eq. (3.23) taken for a single field. It is an interesting question whether a scalar theory exists yielding a result analogous to (3.26) from scattering amplitude arguments.

In part motivated by [15], there have been some attempts to arrive at a SWGC using bound states arguments by somehow introducing short-range repulsive scalar interactions. In order to compare short with long range forces, one needs to fix an energy scale. In [41] a modified version of the RFC was proposed where only the leading interaction is to be compared with gravity. In this way they were able to motivate differential inequalities for the SWGC. A different proposal was made in [37]. They argued against the formation of gravitationally bound states with sizes smaller than their Compton wavelength. This idea was coined as Bound State Conjecture. The latter does not give rise to a differential inequality and it remains non-trivial even when gravity is turned off.

The fact that the PPWGC gives a well defined rationale for the existence of a Scalar Weak Gravity Conjecture is an important result of the present work. So far, it is the only criteria that is translated into a differential constraint including both first and second derivatives of the mass, making direct contact with known $N=2$ BPS constraints, proposing also a generalization to non-SUSY settings. 


\section{Constraints on the scalar potential for moduli}

In the above we have seen how the PPWGC applied to scalars suggests the existence of massive scalars which obey or saturate the bounds, so that gravity is the weakest force. These fields correspond to scalars belonging to BPS multiplets when there is enough SUSY. However, we would like to know whether any constraint may be obtained for other scalars like moduli themselves, once they get a mass. In particular, it would be interesting to see whether the above bounds may give us some constraint on moduli (or other scalars) effective potentials.

One possible connection, inspired by our experience in string theory, is as follows. Moduli $t_{i}$ in string theory are massless classically and get a potential at the quantum level. Such potentials often appear after summing over loop contributions of massive $H_{a}$ particles, like e.g., towers of BPS states. The dependence on massive BPS states may also appear at the non-perturbative level. Those massive particles have masses $m_{a}\left(t_{i}\right)$ which are functions of the moduli already at the classical level, we saw some $N=2$ examples above. In those cases the induced moduli potential will depend on the moduli through the masses of the heavy BPS-like states, $V=V\left(m_{a}\left(t_{i}\right)\right)$. If we insist that the masses of heavy $H_{a}$ scalars $m_{a}$ are subject to the PPSWGC, one might hope to obtain some constraint on the form of the resulting moduli potential. In string theory we typically have plenty of moduli and infinite towers of BPS objects so the task is not easy. Here for simplicity we are going to consider the, admittedly, oversimplified case of a single modulus whose potential is a function of a single massive state $H$ whose mass $m$ obeys a single field version of the constraint eq. (3.7).

In this section we examine whether the scalar WGC leads to constraints similar to those of the dS conjecture. One possible hist of this connection is the appearance in both of the second derivative of the potential. Let us first recall the swampland dS conjecture [25-27] for later comparison. The latter states that the scalar potential for a theory coupled to gravity satisfies either

$$
|\nabla V| \geq c \frac{V}{M_{p}}, \quad \text { or }, \quad \min \left(\nabla_{i} \nabla_{j} V\right) \leq-c^{\prime} \frac{V}{M_{p}^{2}} .
$$

Here $c, c^{\prime}$ are constants of order one. In the second alternative one has the minimum eigenvalue of the Hessian in an orthonormal frame. This refined dS conjecture has the property that dS maxima are allowed (as it should since e.g. the SM has one such maximum) but dS minima are not. This form of the dS conjecture is motivated by arguments which use the covariant entropy bound applied to a dS configuration, see [25-27].

Let us consider for definiteness the case of a $N=1$ supergravity theory with a single modulus $T$. It gets a potential at the quantum level from a massive state with mass $m^{2}\left(T, T^{*}\right)$, so that the modulus potential depends on the moduli only through its dependence on this mass, $V=V\left(m^{2}\left(T, T^{*}\right)\right)$. To simplify notation define $y=m^{2}$. Then it is easy to check that

$$
m_{T}^{2}=\frac{V_{T}}{V_{y}}, \quad m_{\bar{T}}^{2}=\frac{V_{\bar{T}}}{V_{y}} ; \quad m_{T \bar{T}}^{2}=\frac{V_{T \bar{T}}}{V_{y}}-\frac{V_{y y}}{V_{y}}\left(\frac{V_{T} V_{\bar{T}}}{V_{y}^{2}}\right)
$$


Imposing the PPWGC bound in eq. (3.7), one gets the result

$$
g^{T \bar{T}}\left|\frac{V_{T} V_{\bar{T}}}{V_{y}^{2}}\left(1+y \frac{V_{y y}}{V_{y}}\right)-y \frac{V_{T \bar{T}}}{V_{y}}\right| \geq \frac{y^{2}}{M_{p}^{2}} .
$$

If we search for extrema $V_{T}=V_{\bar{T}}=0$ one obtains (assuming $V_{y} \neq 0$ )

$$
g^{T \bar{T}}\left|V_{T \bar{T}}\right| \geq \frac{\left|y V_{y}\right|}{M_{p}^{2}} .
$$

One sees that the second derivative of the potential is bounded below. This is reminiscent of the dS conjecture refinement, that if there is a extremum, the second derivative of the potential must be large enough. However, in the present case it applies both to dS and AdS.

More specific results are obtained if one assumes a power dependence for the potential,i.e., $V=\eta m^{2 \gamma}$, with $\gamma$ a positive number and $\eta= \pm 1$ (see also [38, 39]). Examples of Type IIA orientifolds with fluxes [42-44] scale like $V \sim m^{2}$ at the minima. This behavior is also a prediction of the AdS conjecture in ref. [45], recently tested in e.g. [46-50] within string theory. Another example of this kind of dependence is the case of the ColemanWeinberg one-loop potential, which is proportional to the 4-th power of the mass propagating in the loop. For $V \sim m^{2 \gamma}$ one finds

$$
\left|\left(\frac{\nabla V}{V}\right)^{2}-g^{T \bar{T}} \frac{V_{T \bar{T}}}{|V|}\right| \geq \frac{\gamma}{M_{p}^{2}} .
$$

At extrema one gets the condition

$$
\left|g^{T \bar{T}} V_{T \bar{T}}\right| \geq \gamma \frac{|V|}{M_{p}^{2}}
$$

This constraint is represented in figure 6 . This gives a low-energy bound on the mass of the moduli at the minimum in terms of the value of the potential. It is also somewhat analogous to the refined dS conjecture for $c^{\prime}=\gamma$ and the recent TCC conjecture [51], but it also applies for AdS vacua.

In this case one can see that dS minima are forbidden, at least for potentials such that $V \rightarrow 0$ as $T$ goes to infinity. The point is that, in such a case, if the potential has a $\mathrm{dS}$ minimum, then it must necessarily have a dS local maximum. If the potential is nonsingular, there should be a field path connecting the minimum with the maximum. But, as the figure shows, one cannot continuously go from a minimum to a maximum without going through the forbidden region where gravity is too strong.

It would be interesting to test these minima conditions in the context of the class of Type IIA AdS vacua in refs. [42-44, 46, 47]. A different conjecture also involving also second derivatives of the potential was put forward in [40].

One should take these bounds on potentials with caution. Here we are only considering one modulus with a single massive object verifying PPSWGC constraints, and with a simple potential of the form $V \simeq m^{2 \gamma}$. Still it shows how a possible connection between the dS and scalar WGC conjectures could arise. It would be particularly interesting to generalize, if possible, these arguments to the case of multiple fields. 


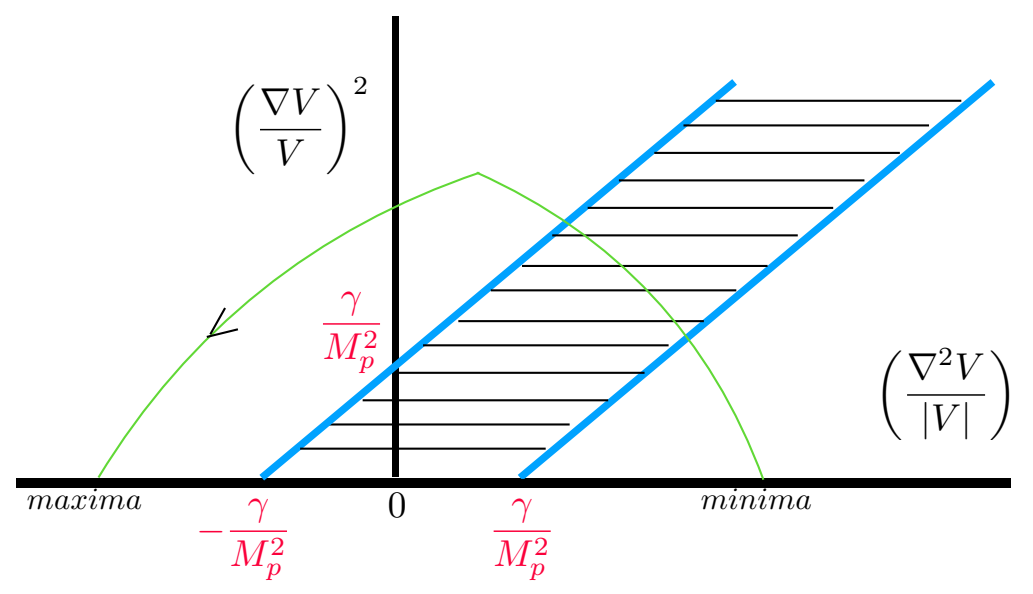

Figure 6. Constraints for the potential of a single modulus when $V \propto m^{2 \gamma}$. In the barred region the gravitational interaction is stronger than the scalar one. If a dS minimum exists, and the potential vanishes at infinity, there must be also a local maximum. This is not possible because going from a minimum to a maximum in field space (green line) one has to go through the forbidden region. The constraint disappears as $M_{p} \rightarrow \infty$.

\section{$5 \quad$ Strong Scalar Weak Gravity Conjecture}

The proposed PPSWGC declares that WGC particles with mass $m$ must exist such that their production rate by massless moduli $\phi$ is constrained as in section 3 . On the other hand, according to the idea that no interaction weaker than gravity exists, one would also expect constraints among interacting scalars. In particular, moduli will in general acquire masses and interactions in the absence of SUSY, and their interactions would be constrained if they must be weaker than gravity. In our previous paper, we named it the strong Scalar Weak Gravity Conjecture $(s S W G C)$. Inspired by the PPSWGC and the scalar constraints found in section 3 one may conjecture for the case of a single self-interacting a sSWGC constraint of the form:

$$
\left|\xi\left(V^{\prime \prime \prime}\right)^{2}-\left(V^{\prime \prime}\right)\left(V^{\prime \prime \prime \prime}\right)\right| \geq \frac{\left(V^{\prime \prime}\right)^{2}}{M_{p}^{2}}
$$

where we have made the replacement $m^{2} \rightarrow V^{\prime \prime}$. Here $\xi$ is some number of order one. In [15] such a constraint was proposed with $\xi=2$, inspired by eq. (3.26). On the other hand eq. (3.29) would suggest instead $\xi=1$. These proposal was not based on a specific set of diagrams but are rather bold generalizations to the case of the self-interactions of a single real scalar field.

One may try to justify a particular value of $\xi$ in terms of the scattering of a couple of real scalars. In this case one would consider the elastic scattering of two scalars almost at rest compared to the threshold production of two scalars from graviton scattering. The diagrams for the scalar scattering are those as in figure 4 and an additional $s$-channel one in which two scalars produce a virtual scalar which then decays into two scalars. Since the mediators are massive, the threshold conditions are $u=t=0$ and also $s=4 m^{2}$. Direct 
application of the Feynman rules give:

$$
N_{1}+N_{2}+N_{3}=-\left(\dot{m}^{2}\right)^{2}\left\{\frac{1}{t-m^{2}}+\frac{1}{u-m^{2}}+\frac{1}{s-m^{2}}\right\}-\ddot{m}^{2},
$$

so one obtains $\xi=5 / 3$. Still, as we will see below, in order to see how masses and parameters are constrained the particular value of $\xi$ is not very relevant, as long as it is of order one.

One important property of the condition (5.1) is that it is a Swampland condition, in the sense that it disappears when gravity decouples. This is unlike the condition without the absolute value put forward in [15]. The constraint in eq. (5.1) passes some interesting tests. It is easy to check that an axion potential of the form $V(\eta)=-M^{4} \cos (\eta / f)$ obeys the constraint as long as the decay constant obeys $f \leq M_{p}$, in agreement with axion WGC arguments [15]. If one considers a Higgs-like potential of the form $V=m_{0}^{2} \phi^{2} / 2+\lambda_{0} \phi^{4} / 4$ ! one gets from eq. (5.1) a constraint

$$
|\lambda|\left|m^{2}(\phi)-\lambda \phi^{2}\right| \geq \xi \frac{m^{4}(\phi)}{M_{p}^{2}},
$$

where $\lambda=\xi \lambda_{0}$ and $m^{2}=V^{\prime \prime}=m_{0}^{2}+\frac{1}{2} \lambda_{0} \phi^{2}$ the field-dependent mass ${ }^{2}$. Note that at small $\phi$ the constraint is verified as long as $\left|\lambda_{0}\right| \geq\left(m_{0} / M_{p}\right)^{2}$, in agreement with Weak Gravity intuitions. The constraints on the plain $m^{2}(\phi)-\lambda \phi^{2}$ are shown in figure 7 for $\lambda=1,0.1$, in units of $M_{p} / \xi^{1 / 2}$. The red area is allowed by the condition. One notices that as the value of the field $\phi \rightarrow 0$ the value of the mass becomes smaller and smaller. This pattern is sharper for smaller $\lambda$. This structure is interesting, as it shows an unexpected field-dependent upper bound on the masses of scalars as the field varies. For values outside the red area the gravitational interaction dominates over the scalar interaction and hence that situation is forbidden. As gravity decouples, the red area covers all the plain.

The sSWGC without the absolute value, for the Higgs-like potential at $\phi=0$ would give $\lambda \leq-\frac{m_{0}^{2}}{M_{p}^{2}}$. Thus for a massive scalar only an unstable potential with $\lambda<0$ would be allowed. Based on this observation (as applied to the case in [15]) some counter-examples were argued to exist in [37]. In the new results in the present paper both signs of $\lambda$ are allowed. In this regard note that a constraint without the absolute value, would also forbid field ranges with $|\phi|^{2} \leq 2 m_{0}^{2} / \lambda$, even in the absence of gravity. Such a forbidden field range is no longer present in the new constraints eq. (5.1). Note that somewhat analogous results, in particular, the presence of an absolute value and the coefficient $\frac{5}{3}$ were also obtained in [41], although starting from different physical principles.

As we said, as $\lambda \rightarrow 0$ the condition is violated. It was already pointed out in [15] that, since $\lambda$ in the SM vanish at a high scale $\simeq 10^{10}-10^{13} \mathrm{GeV}$, new physics is predicted to appear at this scale, since the quartic interaction becomes weaker than gravity. An elegant solution to this problem is that SUSY is recovered below that scale, getting a theory consistent with quantum gravity. Note that this behaviour appears only in the presence of gravity and hence would be a Swampland constraint, not a field theory constraint.

The possibility of a cancellation between trilinear and quartic contributions is more general than the above constraint, and appear in other examples due to the structure of the 

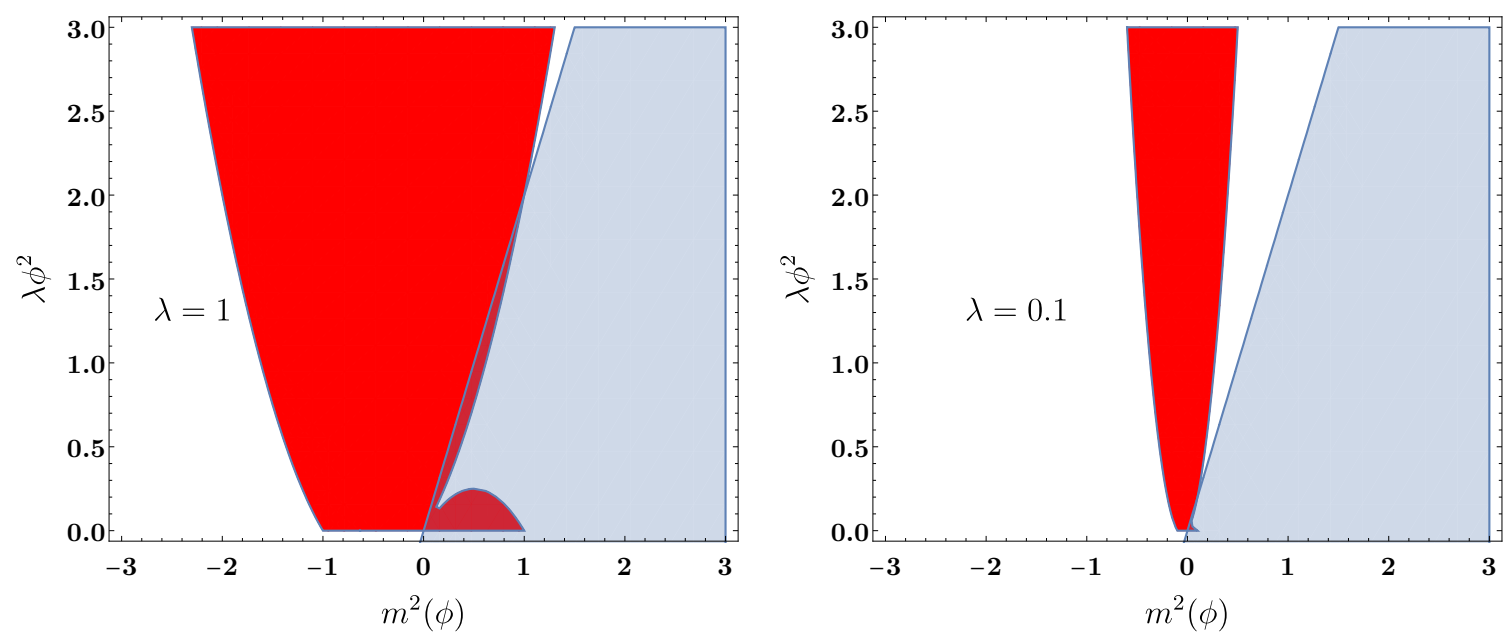

Figure 7. Constraints on the $m^{2}(\phi)-\lambda \phi^{2}$ plane for a quartic real scalar potential for fixed $\lambda=1$ and 0.1 in $M_{p} / \xi^{1 / 2}$ units. The red region is allowed. The blue region marks when $m_{0}^{2}>0$. As the field $\phi$ tends to zero, the field-dependent mass $m^{2}(\phi)$ also decreases. The allowed region also gets narrower as $\lambda$ decreases. When gravity decouples the allowed red region covers all the upper half-plain.

amplitude in eq. (3.5). In fact this is the origin of the forbidden bands in figures 5, 6. This may lead to potential inconsistencies with the scalar WGC at finite points in moduli space in some examples, indicating their inconsistency or incompleteness if gravity is present. In the $N=2$ SUSY examples shown above this does not happen for the BPS states, which lie at the boundary of the forbidden regions. But it may happen e.g. in non-SUSY examples for some field value. Turning the argument around, the presence of these forbidden regions in non-SUSY theories coupled to gravity could be an argument for the presence of SUSY at some scale in the low energy effective action.

It is important to remark that the sSWGC stands on a less firm ground than the general PPWGC or the SWGC discussed in sections 2 and 3. In particular it is not obvious that the simple recipe $m^{2} \rightarrow V^{\prime \prime}$ in the PPWGC constraints is sufficiently justified. To our knowledge, there is however no counterexample to the sSWGC here considered with an absolute value included. It would be interesting to find further support for generalized SWGC like this. If one takes a constraint like eq. (5.1) to be valid for any single scalar potential, there are important phenomenological implications, as already shown for the old version of the constraint in [15]. In addition, it would also be interesting to find a multi-field generalization of these constraints.

\section{$6 \quad$ Final comments and conclusions}

In this paper we have proposed pair production of massive particles at threshold as a means to compare the gravitational to the gauge and scalar interactions. Equivalent results would be obtained from pair annihilation of massive particles into photons/gravitons/moduli. Imposing that the production rates from gravitons is always smaller than that from gauge bosons or moduli gives rise to specific WGC constraints. In the case of U(1) interactions 
this diagrammatic prescription reproduces the same results as obtained from instability of extremal black-holes. On the other hand when applied to pair creation from moduli, a scalar WGC constraint depending on first and second derivatives of the mass appear. Intriguingly, imposing saturation of the conditions one obtains simple differential equations. Some of the solutions match with known results in $N=2$ BPS examples and are consistent with the Swampland Distance Conjecture. Other solutions have more general asymptotic behaviour.

One interesting aspect of this approach is that it derives the $\mathrm{U}(1)^{n}$ WGC conjectures and a scalar WGC from the same general principle of gravity being the weakest interaction. The form of the scalar WGC depends on whether we are dealing with complex or real moduli and the metric in moduli space. For the case of $n$ complex moduli the constraint eq. (3.7) is obtained. One has to view our proposal as complementary to the constraints obtained from extremal black-hole instability and the Repulsive conjectures. We think our proposal is particularly interesting in its application to obtain constraints on scalar couplings.

One point to note is that our condition is a quantum relativistic condition since it involves particle production and interaction rates rather than amplitudes. This is unlike the case of one photon/graviton exchange with particles at rest which give rise to the classical non-relativistic Coulomb/Newton potentials. The presence of rates (absolute values of amplitudes) plays also an important role in the emergence of duality symmetries among the states saturating the bounds. It is particularly remarkable how the existence of momenta and winding (extended objects) emerges from simple scattering amplitude considerations in the effective low-energy theory.

There are many aspects which deserve further study. One interesting question is the applicability of the constraints of scalar moduli in non-SUSY theories, in which a moduli space of massless moduli does not in general exist. In this connection, some of the saturating solutions for the scalar WGC constraints that we obtain may be interpreted as the bosonic subsector of BPS and special geometry conditions in $N=2$ supergravity theory. On the other hand we believe that the principle of gravity being the weakest force is independent of supersymmetry and one can expect that the constraints will still apply at least in theories with spontaneously broken SUSY.

It would be interesting to test the condition eq. (3.7) in specific string settings, like the towers of BPS states getting massless at large moduli in Type IIA and Type IIB CY compactifications, as in refs. [17-22]. Another interesting direction for further research would include the extension to higher dimensions and to non-Abelian gauge groups. It would also be important to obtain constraints on scalar potentials of moduli and other scalar fields along the lines of sections 4 (eq. (4.6)) and 5 in this paper and study its implications in cosmology and particle physics.

It is important to determine what is it exactly that goes wrong if the PPWGC condition is violated. Pair production of charged particles is a characteristic of black-hole radiation and it would be important to elucidate the precise connection, if at all, of the present ideas with black-hole physics. Our conditions also imply that the annihilation rate of charged black-holes into photons must be larger than to gravitons. Perhaps the PPWGC constraints appear as additional dynamical requirements. It would be interesting to extend the results of this work by considering pair production of particles in backgrounds different from flat space-time, not only in the context of black-holes but also in AdS and $d S$. 
More generally, we would like to understand whether and why gravity should be weaker than any other interaction, and the role of this condition in the general context of Quantum Gravity and String Theory.

\section{Acknowledgments}

We thank G. Aldazabal, A. Font, A. Herráez, F. Marchesano, M. Montero, A. Uranga, and I. Valenzuela for useful discussions. This workis is supported by the Spanish Research Agency (Agencia Española de Investigación) through the grants IFT Centro de Excelencia Severo Ochoa SEV-2016-0597, the grant GC2018-095976-B-C21from MCIU/AEI/FEDER, UE and the grant PA2016-78645-P. E.G. is supported by the Spanish FPU Grant No. FPU16/03985.

Open Access. This article is distributed under the terms of the Creative Commons Attribution License (CC-BY 4.0), which permits any use, distribution and reproduction in any medium, provided the original author(s) and source are credited.

\section{References}

[1] C. Vafa, The String landscape and the swampland, hep-th/0509212 [INSPIRE].

[2] N. Arkani-Hamed, L. Motl, A. Nicolis and C. Vafa, The String landscape, black holes and gravity as the weakest force, JHEP 06 (2007) 060 [hep-th/0601001] [INSPIRE].

[3] H. Ooguri and C. Vafa, On the Geometry of the String Landscape and the Swampland, Nucl. Phys. B 766 (2007) 21 [hep-th/0605264] [INSPIRE].

[4] E. Palti, The Swampland: Introduction and Review, Fortsch. Phys. 67 (2019) 1900037 [arXiv: 1903.06239] [INSPIRE].

[5] C. Cheung and G.N. Remmen, Naturalness and the Weak Gravity Conjecture, Phys. Rev. Lett. 113 (2014) 051601 [arXiv: 1402 .2287] [INSPIRE].

[6] B. Heidenreich, M. Reece and T. Rudelius, Sharpening the Weak Gravity Conjecture with Dimensional Reduction, JHEP 02 (2016) 140 [arXiv:1509.06374] [INSPIRE].

[7] B. Heidenreich, M. Reece and T. Rudelius, Evidence for a sublattice weak gravity conjecture, JHEP 08 (2017) 025 [arXiv:1606.08437] [INSPIRE].

[8] M. Montero, G. Shiu and P. Soler, The Weak Gravity Conjecture in three dimensions, JHEP 10 (2016) 159 [arXiv:1606.08438] [INSPIRE].

[9] S. Andriolo, D. Junghans, T. Noumi and G. Shiu, A Tower Weak Gravity Conjecture from Infrared Consistency, Fortsch. Phys. 66 (2018) 1800020 [arXiv: 1802.04287] [INSPIRE].

[10] B. Heidenreich, M. Reece and T. Rudelius, Repulsive Forces and the Weak Gravity Conjecture, JHEP 10 (2019) 055 [arXiv:1906.02206] [INSPIRE].

[11] E. Palti, The Weak Gravity Conjecture and Scalar Fields, JHEP 08 (2017) 034 [arXiv: 1705.04328] [INSPIRE].

[12] D. Lüst and E. Palti, Scalar Fields, Hierarchical UV/IR Mixing and The Weak Gravity Conjecture, JHEP 02 (2018) 040 [arXiv:1709.01790] [INSPIRE]. 
[13] G. Dall'Agata and M. Morittu, Covariant formulation of BPS black holes and the scalar weak gravity conjecture, JHEP 03 (2020) 192 [arXiv:2001.10542] [INSPIRE].

[14] B.R. Holstein, Graviton Physics, Am. J. Phys. 74 (2006) 1002 [gr-qc/0607045] [InSPIRE].

[15] E. Gonzalo and L.E. Ibáñez, A Strong Scalar Weak Gravity Conjecture and Some Implications, JHEP 08 (2019) 118 [arXiv: 1903.08878] [INSPIRE].

[16] S.-J. Lee, W. Lerche and T. Weigand, A Stringy Test of the Scalar Weak Gravity Conjecture, Nucl. Phys. B 938 (2019) 321 [arXiv:1810.05169] [INSPIRE].

[17] N. Gendler and I. Valenzuela, Merging the Weak Gravity and Distance Conjectures Using BPS Extremal Black Holes, arXiv: 2004.10768 [INSPIRE].

[18] T.W. Grimm, E. Palti and I. Valenzuela, Infinite Distances in Field Space and Massless Towers of States, JHEP 08 (2018) 143 [arXiv:1802.08264] [INSPIRE].

[19] P. Corvilain, T.W. Grimm and I. Valenzuela, The Swampland Distance Conjecture for Kähler moduli, JHEP 08 (2019) 075 [arXiv: 1812.07548] [INSPIRE].

[20] A. Font, A. Herráez and L.E. Ibáñez, The Swampland Distance Conjecture and Towers of Tensionless Branes, JHEP 08 (2019) 044 [arXiv: 1904.05379] [INSPIRE].

[21] S.-J. Lee, W. Lerche and T. Weigand, Tensionless Strings and the Weak Gravity Conjecture, JHEP 10 (2018) 164 [arXiv:1808.05958] [INSPIRE].

[22] S.-J. Lee, W. Lerche and T. Weigand, Modular Fluxes, Elliptic Genera, and Weak Gravity Conjectures in Four Dimensions, JHEP 08 (2019) 104 [arXiv:1901.08065] [INSPIRE].

[23] S.-J. Lee, W. Lerche and T. Weigand, Emergent Strings from Infinite Distance Limits, arXiv: 1910.01135 [INSPIRE].

[24] E. Gonzalo, L.E. Ibáñez and A.M. Uranga, Modular Symmetries and the Swampland Conjectures, JHEP 05 (2019) 105 [arXiv: 1812.06520] [INSPIRE].

[25] G. Obied, H. Ooguri, L. Spodyneiko and C. Vafa, de Sitter Space and the Swampland, arXiv: 1806.08362 [INSPIRE].

[26] S.K. Garg and C. Krishnan, Bounds on Slow Roll and the de Sitter Swampland, JHEP 11 (2019) 075 [arXiv: 1807.05193] [inSPIRE].

[27] H. Ooguri, E. Palti, G. Shiu and C. Vafa, Distance and de Sitter Conjectures on the Swampland, Phys. Lett. B $\mathbf{7 8 8}$ (2019) 180 [arXiv:1810.05506] [INSPIRE].

[28] B. Heidenreich, The Weak Gravity Conjecture and Repulsive Forces, talk at String Phenomenology 2019, CERN, 24-28 June 2019 [https://indico.cern.ch/event/782251/contributions/3441855/].

[29] S.Y. Choi, J.S. Shim and H.S. Song, Factorization in graviton interactions, Phys. Rev. D 48 (1993) 5465 [hep-ph/9310259] [INSPIRE].

[30] S.Y. Choi, J.S. Shim and H.S. Song, Factorization of gravitational Compton scattering amplitude in the linearized version of general relativity, Phys. Rev. D 48 (1993) 2953 [hep-ph/9306250] [INSPIRE].

[31] B.R. Holstein, Factorization in graviton scattering and the 'natural' value of the g-factor, gr-qc/0607058 [INSPIRE].

[32] Z. Bern, Perturbative quantum gravity and its relation to gauge theory, Living Rev. Rel. $\mathbf{5}$ (2002) 5 [gr-qc/0206071] [INSPIRE]. 
[33] J.J.M. Carrasco, Gauge and Gravity Amplitude Relations, in Theoretical Advanced Study Institute in Elementary Particle Physics: Journeys Through the Precision Frontier: Amplitudes for Colliders, pp. 477-557, WSP (2015) [DOI] [arXiv: 1506.00974] [INSPIRE].

[34] L.E. Ibáñez and A.M. Uranga, String theory and particle physics: An introduction to string phenomenology, Cambridge University Press (2012) [INSPIRE].

[35] A. Ceresole, R. D'Auria, S. Ferrara and A. Van Proeyen, Duality transformations in supersymmetric Yang-Mills theories coupled to supergravity, Nucl. Phys. B 444 (1995) 92 [hep-th/9502072] [INSPIRE].

[36] S. Bellucci, S. Ferrara and A. Marrani, Attractors in Black, Fortsch. Phys. 56 (2008) 761 [arXiv: 0805.1310] [INSPIRE].

[37] B. Freivogel, T. Gasenzer, A. Hebecker and S. Leonhardt, A Conjecture on the Minimal Size of Bound States, SciPost Phys. 8 (2020) 058 [arXiv: 1912.09485] [INSPIRE].

[38] L.E. Ibáñez, On Towers and Scalars, talk at String Phenomenology 2019, CERN, 24-28 June 2019, [https://indico.cern.ch/event/782251/contributions/3441881/].

[39] D. Andriot, N. Cribiori and D. Erkinger, The web of swampland conjectures and the TCC bound, JHEP 07 (2020) 162 [arXiv: 2004.00030] [inSPIRE].

[40] D. Andriot and C. Roupec, Further refining the de Sitter swampland conjecture, Fortsch. Phys. 67 (2019) 1800105 [arXiv: 1811.08889] [INSPIRE].

[41] K. Benakli, C. Branchina and G. Lafforgue-Marmet, Revisiting the Scalar Weak Gravity Conjecture, Eur. Phys. J. C 80 (2020) 742 [arXiv:2004.12476] [InSPIRE].

[42] O. DeWolfe, A. Giryavets, S. Kachru and W. Taylor, Type IIA moduli stabilization, JHEP 07 (2005) 066 [hep-th/0505160] [INSPIRE].

[43] P.G. Cámara, A. Font and L.E. Ibáñez, Fluxes, moduli fixing and MSSM-like vacua in a simple IIA orientifold, JHEP 09 (2005) 013 [hep-th/0506066] [INSPIRE].

[44] G. Aldazabal, P.G. Cámara, A. Font and L.E. Ibáñez, More dual fluxes and moduli fixing, JHEP 05 (2006) 070 [hep-th/0602089] [INSPIRE].

[45] D. Lüst, E. Palti and C. Vafa, AdS and the Swampland, Phys. Lett. B 797 (2019) 134867 [arXiv: 1906.05225] [INSPIRE].

[46] R. Blumenhagen, M. Brinkmann and A. Makridou, Quantum Log-Corrections to Swampland Conjectures, JHEP 02 (2020) 064 [arXiv:1910.10185] [INSPIRE].

[47] A. Font, A. Herráez and L.E. Ibáñez, On scale separation in type-II AdS flux vacua, JHEP 03 (2020) 013 [arXiv: 1912.03317] [INSPIRE].

[48] D. Junghans, O-Plane Backreaction and Scale Separation in Type IIA Flux Vacua, Fortsch. Phys. 68 (2020) 2000040 [arXiv:2003.06274] [INSPIRE].

[49] G. Buratti, J. Calderon, A. Mininno and A.M. Uranga, Discrete Symmetries, Weak Coupling Conjecture and Scale Separation in AdS Vacua, JHEP 06 (2020) 083 [arXiv:2003.09740] [INSPIRE].

[50] F. Marchesano, E. Palti, J. Quirant and A. Tomasiello, On supersymmetric AdS 4 orientifold vacua, JHEP 08 (2020) 087 [arXiv:2003.13578] [INSPIRE].

[51] A. Bedroya and C. Vafa, Trans-Planckian Censorship and the Swampland, JHEP 09 (2020) 123 [arXiv: 1909.11063] [INSPIRE]. 